\title{
Enhanced sensitization to animal, interpersonal, and intergroup fear-relevant stimuli (but no evidence for selective one-trial fear learning)
}

\section{OTTMAR V. LIPP, ${ }^{\mathrm{a}, \mathrm{b}}$ SOPHIE L. CRONIN, ${ }^{\mathrm{c}}$ SAKINAH S. J. ALHADAD ${ }^{\mathrm{c}}{ }^{\text {AND }}$ CAMILLA C. LUCK ${ }^{\mathrm{a}, \mathrm{b}}$}

${ }^{a}$ School of Psychology and Speech Pathology, Curtin University, Perth, Australia

${ }^{\mathrm{b}}$ ARC-SRI: Science of Learning Research Centre, Brisbane, Australia

'School of Psychology, The University of Queensland, St. Lucia, Australia

\begin{abstract}
Selective sensitization has been proposed as an alternative explanation for enhanced responding to animal fear-relevant stimuli-snakes and spiders-during extinction of Pavlovian fear conditioning. The current study sought to replicate the phenomenon using a shock workup procedure as the sensitizing manipulation and to extend it to interpersonal and intergroup fear-relevant stimuli-angry faces and other-race faces. Assessment of selective sensitization was followed by a one-trial fear learning procedure. Selective sensitization, larger electrodermal responses to fear-relevant than to control stimuli after sensitization, or a larger increase in electrodermal responding to fear-relevant than to control stimuli after sensitization was observed across stimulus domains. However, the one-trial fear learning procedure failed to provide evidence for enhanced fear conditioning to fear-relevant stimuli. One-trial fear learning was either absent or present for fear-relevant and nonfear-relevant stimuli. The current study confirms that electrodermal responses to fearrelevant stimuli across stimulus domains are subject to selective sensitization.
\end{abstract}

Descriptors: Fear learning, Fear relevance, Electrodermal responses, Animal fear, Interpersonal fear, Intergroup fear

Past research in human fear learning has provided considerable support for the notion that associations between some conditional stimuli and aversive outcomes are more resistant to extinction than are others (for recent reviews, see Mallan, Lipp, \& Cochrane, 2013; Öhman, 2009). This enhanced resistance to extinction has been shown for fear conditioned to animal fear-relevant stimulipictures of snakes and spiders (Öhman, Fredrikson, Hugdahl, \& Rimmö, 1976), interpersonal fear-relevant stimuli-pictures of angry faces (Öhman \& Dimberg, 1978), and intergroup fearrelevant stimuli-pictures of other-race faces (Olsson, Ebert, Banaji, \& Phelps, 2005). There is, however, currently no agreement as to the process that underlies this resistance to extinction or whether the resistance to extinction across different stimulus domains reflects a single (Öhman, 2009) or different underlying processes (Mallan et al., 2013). This largely reflects a shortage of studies that assess the effects of the same experimental manipulations on fear learning across different stimulus domains.

Evolutionary-based preparedness was the first account proposed to explain why some associations are more resistant to extinction

This work was supported by grant numbers DP120100750 and SR120300015 from the Australian Research Council.

Address correspondence to: Ottmar V. Lipp, Ph.D., Curtin University, School of Psychology and Speech Pathology, Perth, WA, 6845, Australia. E-mail: ottmar.lipp@curtin.edu.au than others (Seligman, 1971). Reviewing the characteristics that distinguish phobic fear from conditioned fear, which is readily extinguished, Seligman formulated a set of criteria that differentiate prepared learning from nonprepared Pavlovian conditioning. Prepared learning is said to be (a) acquired fast and resistant to extinction; (b) selective to specific parings of conditional and unconditional stimuli (i.e., preparedness is a characteristic of associations, not of particular stimuli); (c) subject to one-trial learning; and (d) encapsulated from cognition. Although one may argue that the preparedness account does not provide more than a post hoc description of phobic fear, it has the advantage of providing explicit criteria that permit the distinction of prepared from nonprepared learning.

Past research on fear conditioned to animal fear-relevant stimuli has yielded results that are consistent with the criteria posed by prepared learning theory. However, alternative accounts have also been developed to explain one key finding: enhanced resistance to extinction of fear conditioned to fear-relevant stimuli. Bond and Siddle (1986) proposed differential preexposure to fear-relevant and nonfear-relevant stimuli as a potential explanation, whereas Davey (1992) proposed an expectancy model of preparedness effects. Lovibond, Siddle, and Bond (1993) proposed selective sensitization as a nonassociative account for this phenomenon. This proposal contends that enhanced responding during extinction to a fear-relevant $\mathrm{CS}^{+}$, a conditional stimulus that had been paired with an aversive unconditional stimulus, does not reflect the fear 
conditioned during acquisition, but the general capacity of fearrelevant conditional stimuli to elicit enhanced responses under conditions of threat. Selective sensitization is said to affect the results, although studies of prepared learning attempt to control for effects of a priori differences in response elicitation by using a differential conditioning design in which two fear-relevant conditional stimuli are presented, one paired with the unconditional stimulus $\left(\mathrm{CS}^{+}\right)$ and one presented alone $\left(\mathrm{CS}^{-}\right)$. This differential design is said to control for nonassociative factors that affect responding, as these should enhance responses to $\mathrm{CS}^{+}$and $\mathrm{CS}^{-}$equally, whereas the difference in responding to the two conditional stimuli provides a measure of associative learning.

Lovibond et al. (1993) provided support for the selective sensitization account by showing enhanced electrodermal responding to a fear-relevant stimulus relative to a nonfear-relevant stimulus after fear conditioning, even though neither stimulus had been paired with shock. In contrast, there was no difference between a second pair of fear-relevant and nonfear-relevant stimuli after they had served as $\mathrm{CS}^{-}$in a differential conditioning procedure. This pattern was interpreted to mean that training a fear-relevant stimulus as a $\mathrm{CS}^{-}$eliminates its propensity for enhanced electrodermal responding. In Experiment 2, Lovibond et al. demonstrated that training a fear-relevant stimulus as a signal for danger $\left(\mathrm{CS}^{+}\right)$does not attenuate its capacity to elicit enhanced responses. Thus, the larger differential responding to fear-relevant relative to nonfear-relevant $\mathrm{CS}^{+}$ and $\mathrm{CS}^{-}$observed during extinction training (or differential responding across a larger number of extinction trials) is explained as selective sensitization, which is attenuated for the fear-relevant $\mathrm{CS}^{-}$after differential training as a safety signal, rather than as a slower extinction of fear conditioned to the fear-relevant $\mathrm{CS}^{+}$. Although the selective sensitization account is not clear as to how the prepotency of a stimulus is acquired (Lovibond et al., 1993, favor a noncognitive genetic model whereas Lovibond, Hanna, Siddle, \& Bond, 1994, suggested a cognitive model), it offers an intriguing alternative to the preparedness account (see Öhman \& Mineka, 2001, for a discussion).

Selective sensitization is well documented in research employing animal fear-relevant stimuli (Lovibond et al., 1994; Öhman, Eriksson, Fredriksson, Hugdahl, \& Olofsson, 1974; for a review, see Öhman \& Mineka, 2001) and has been shown for ontogenetic fear-relevant stimuli (guns) as well (Hugdahl \& Johnsen, 1989; Lovibond et al., 1994). It is currently unclear, however, whether interpersonal and intergroup fear-relevant stimuli are also subject to selective sensitization. Although not designed explicitly to do this, studies of prepared learning that include a habituation phase prior to fear conditioning offer an opportunity to provide evidence for the selective sensitization account. These habituation phases include the presentation of fear-relevant and nonfear-relevant stimuli, either to the same or different participants. Moreover, most involve a shock workup procedure in which the intensity of the unconditional stimulus is determined individually. This procedure should be sufficient to elicit sensitization, which in turn should selectively enhance responding to the fear-relevant stimuli presented during the habituation phase. A brief, selective review of the relevant studies that presented interpersonal and intergroup fearrelevant stimuli in a habituation phase after shock workup provides only sparse support for selective sensitization, defined here as larger electrodermal responses to the fear-relevant than the nonfear-relevant control stimuli. Of 11 studies employing angry (a) or fearful (f) faces as interpersonal fear-relevant stimuli, eight did not find differences between fear-relevant and control expressions, happy (h) or neutral (n) (Esteves, Dimberg, \& Öhman, 1994 [a vs. h]; Lanzetta \& Orr, 1980 [f vs. h]; Lanzetta \& Orr, 1981 [f vs. h, n]; Mazurski, Bond, Siddle, \& Lovibond, 1996 [a vs. h]; Öhman \& Dimberg, 1978 [a vs. h]; Parra, Esteves, Flykt, \& Öhman, 1997 [a vs. h, n]; Pitman \& Orr, 1986 [a vs. n]; Rowles, Lipp, \& Mallan, 2012 [a vs. h]), whereas three did (Hamm, Vaitl, \& Lang, 1989 [a vs. landscape pictures]; Orr \& Lanzetta, 1980 [f vs. h-marginal difference]; Bramwell, Mallan, \& Lipp, 2014 [a vs. h]). Two studies of fear conditioned to other-race faces failed to find differences in electrodermal responding to Caucasian and African American faces during habituation (Bramwell et al., 2014; Olsson et al., 2005); however, Bramwell et al. reported faster habituation to ingroup faces. This rather limited evidence may reflect the fact that these studies were not designed to test for the presence of selective sensitization.

The present study was designed to assess explicitly whether selective sensitization can be observed for animal, interpersonal, and intergroup fear-relevant stimuli using a constant procedure across experiments. To maximize the sensitivity of the design, a within-subject design was used in which the same participants were presented with two fear-relevant and two nonfear-relevant stimuli (Olsson et al., 2005). Participants were presented with a brief habituation sequence followed by a shock workup and a repetition of the habituation series. Assessment of selective sensitization was followed by an assessment of one-trial fear learning, which was added opportunistically as (a) there are only few studies that extended on the initial report that one-trial fear learning is limited to animal fear-relevant stimuli (Öhman et al., 1975), and (b) to comply with the instruction that "electrotactile unconditional stimuli might be presented," which was given to participants after the shock workup and before the beginning of the second habituation sequence. One-trial learning is one of the criteria for prepared learning formulated by Seligman (1971), but has been assessed only infrequently in prior research. The entire experimental session was preceded and followed by a rating phase in which participants were asked to rate the pleasantness of the conditional stimuli used. This was included to expose participants to the stimuli used prior to the first habituation sequence and to obtain a second, evaluative index of fear learning.

\section{Experiment 1}

\section{Method}

Participants. Twenty-five undergraduate students ( 8 males) aged between 18 and 33 years (mean age: 22.88 years) volunteered in exchange for course credit and provided informed consent. The research protocol was approved by the University of Queensland ethics review board.

Apparatus. Eight colored images of animals, two snakes, two spiders, two fish, and two birds (Ho \& Lipp, 2014), were used as conditional stimuli (CS). Each participant was presented with only a subset of four pictures (one each of snake, spider, fish, and bird), and stimulus sets were counterbalanced across participants. All stimuli were $520 \times 390$ pixels in size and presented centrally against a black background for $6 \mathrm{~s}$. The unconditional stimulus (US), a 200-ms electrotactile stimulation, pulsed at $50 \mathrm{~Hz}$ and generated by a Grass SD9 stimulator, was applied via a concentric electrode attached to the middle of the dominant anterior forearm. The experiment was controlled using DMDX software (Forster \& Forster, 2003). 
Electrodermal activity was recorded using two 8-mm $\mathrm{Ag} / \mathrm{AgCl}$ electrodes filled with an isotonic electrolyte and attached to the thenar and hypothenar eminences of the nondominant hand. Each participant's respiration rate was monitored with a respiratory effort transducer attached with an adjustable Velcro strap around the lower torso. All physiological responses were recorded with a Biopac MP150 system at $1000 \mathrm{~Hz}$.

Procedure. Upon arrival at the laboratory, participants were seated in a separate cubicle in front of a computer screen and provided informed consent. The experimental procedure was explained to them and measurement devices for electrodermal activity and respiration were attached. After a 3-min baseline recording during which participants were asked to relax without falling asleep, participants were presented with the four pictures used in their stimulus sequence and asked to rate their pleasantness on a 9-point Likert scale. The text displayed on the screen read: "Please rate on a scale of 1 to 9 , where $1=$ unpleasant and $9=$ pleasant." After this initial pre-rating, participants were instructed to remain relaxed and attentive while the first habituation sequence comprising four presentations of each of the four pictures was presented. After completion of the first habituation sequence, the experimenter entered the participant's cubicle and attached the shock electrodes. Participants underwent a shock workup, during which they adjusted the intensity of the electrotactile stimulation to a level that they found "unpleasant, but not painful." The shock workup commenced for all participants with the presentation of a stimulus of an intensity of $0 \mathrm{~V}$. Stimulus intensity was increased in steps of $10 \mathrm{~V}$ until the participant reported a sensation, usually described as a light tingle. Intensity was then increased in steps of $5 \mathrm{~V}$ until the participants described the intensity as unpleasant, but not painful. The final physical stimulus intensity and number of stimuli received differed across participants as the aim was to match the sensation caused by the electrotactile stimulus across participants. Physical stimulus intensities ranged from 25 to $60 \mathrm{~V}$.

Following the shock workup, participants then received instructions to remain relaxed and attentive and were presented with a second habituation sequence, followed by acquisition and extinction phases without interruption. The stimulus sequence used in the second habituation sequence was the same as the first. The acquisition phase consisted of one presentation of each of the four pictures. Offset of one fear-relevant CS, (e.g., the snake) and one nonfearrelevant CS (e.g., the bird) coincided with the onset of the electrotactile stimulus $\left(\mathrm{CS}^{+}\right)$, whereas the remaining pictures (e.g., the spider and the fish) were presented alone $\left(\mathrm{CS}^{-}\right)$. The nature of the $\mathrm{CS}^{+}$and $\mathrm{CS}^{-}$, and the presentation order, were counterbalanced across participants. The extinction sequence comprised six presentations of each picture structured in the same manner as the habituation sequence. No electrotactile stimuli were presented during extinction. Intertrial intervals varied randomly between 18 and $22 \mathrm{~s}$ (CS offset to CS onset) in each phase.

The trials were arranged in a pseudorandom sequence for all phases (first and second habituation, acquisition, and extinction) with the restrictions that no more than two consecutive trials were the same and at the beginning of each phase all pictures had been shown before one was repeated. The stimulus sequence was counterbalanced across participants based on three factors: the particular animal image used as $\mathrm{CS}^{+}$and $\mathrm{CS}^{-}$; the nature of the $\mathrm{CS}\left(\mathrm{CS}^{+}\right.$or $\mathrm{CS}^{-}$) presented first during habituation/acquisition/extinction, and the nature of the animal stimulus (fear-relevant or nonfear-relevant) presented first during habituation/acquisition/extinction. As a result, eight different trial sequences were developed such that the average serial position of the different pictures was the same across sequences. After completion of the extinction sequence, participants provided a second postexperimental rating of the stimuli presented during the experiment and completed a contingency questionnaire. The questionnaire presented the four animal images used in the experiment in a randomized order and asked participants to indicate which animals were paired with a shock. A debriefing session was conducted upon completion of the questionnaire.

Response definition and scoring. Skin conductance response magnitudes were scored throughout the experiment. During the habituation phases, first-interval responses were scored as the largest response that began 1-4 s after CS onset. Electrodermal firstinterval responses elicited by stimulus onset provide an indicator of orienting (Öhman, 1983). During acquisition, electrodermal response magnitudes were scored in the latency interval of $1-4 \mathrm{~s}$ after unconditional stimulus onset to yield a measure of unconditional responding. During extinction, electrodermal first- and second-interval responding were scored. Second-interval responses were scored in the latency interval of 4-7 s after CS onset to provide an additional measure of unconditional stimulus anticipation (Prokasy \& Kumpfer, 1973).

Prior to analysis, skin conductance responses were square root transformed to reduce the impact of skew and range corrected to reduce the effects of individual differences. The range correction was obtained by dividing each response by the maximum response produced by a participant, typically that to the first or the second unconditional stimulus presentation during acquisition.

Statistical analyses. Electrodermal first-interval responses from the two habituation phases were averaged into blocks of two trials across the two exemplars within the two fear relevance categories and subjected to a $2 \times 2 \times 4$ (Fear relevance [fear-relevant vs. nonfear-relevant] $\times$ Phase [preworkup vs. postworkup] $\times$ Block [B1-B4]) factorial repeated measures analyses of variance (ANOVAs). This omnibus analysis was complemented by two targeted analyses. The first compared electrodermal orienting to the initial presentations of the fear-relevant and nonfear-relevant animal pictures during the first habituation sequence using a within-subject $t$ test. The second assessed the effect of the shock workup on responding to the fear-relevant and nonfear-relevant animal pictures in a $2 \times 2$ (Fear Relevance [fear-relevant vs. nonfear-relevant] $\times$ Block [last block of first habituation vs. first block of second habituation]) factorial repeated measures ANOVA.

Electrodermal unconditional responses were compared with a within-subject $t$ test whereas electrodermal first- and secondinterval responses during extinction were subjected to independent $2 \times 2 \times 3$ (Fear Relevance [fear-relevant vs. nonfear-relevant] $\times$ $\mathrm{CS}\left[\mathrm{CS}^{+}\right.$vs. $\left.\mathrm{CS}^{-}\right] \times$Block [E1-E3]) factorial repeated measures ANOVAs. Pre- and postexperimental rating data were analyzed in a $2 \times 2 \times 2$ (Fear Relevance [fear-relevant vs. nonfear-relevant] $\times$ $\mathrm{CS}\left[\mathrm{CS}^{+}\right.$vs. $\left.\mathrm{CS}^{-}\right] \times$Phase [preexperimental vs. postexperimental]) factorial repeated measures ANOVAs.

\section{Results}

Electrodermal data. The upper panel of Figure 1 displays the electrodermal first-interval responses recorded during the two habituation phases. Electrodermal responses did not differ between fear-relevant and nonfear-relevant stimuli and declined during the first habituation phase. As expected, the shock workup procedure 

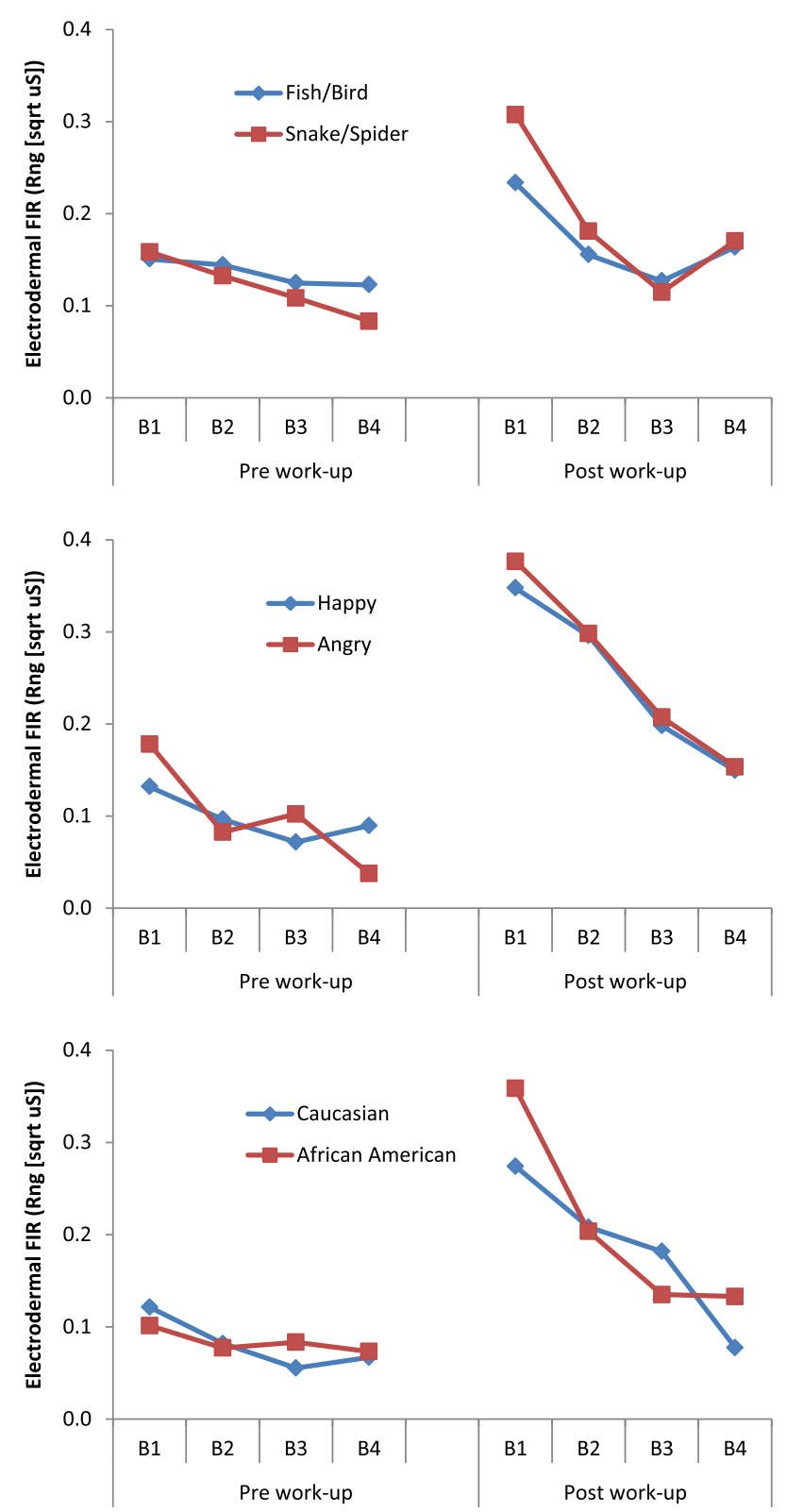

Figure 1. Mean electrodermal first-interval responses to fear-relevant and nonfear-relevant stimuli as a function of trial blocks during habituation sequences presented before and after shock workup in Experiment 1 (upper), Experiment 2 (middle), and Experiment 3 (lower).

resulted in increased responses to both classes of animals, with a larger increase for fear-relevant animals. Subsequent habituation was fast and did not differ between stimuli. The omnibus ANOVA yielded a main effect for block, $F(3,22)=7.67, p=.001$, $\eta_{\mathrm{p}}^{2}=.860$.

Electrodermal orienting at the beginning of the first habituation sequence did not differ between fear-relevant, $M=0.159$, $S D=0.182$, and nonfear-relevant stimuli, $M=0.151, S D=0.243$, $t(24)=0.169, p=.867$. The ANOVA assessing the effects of sensitization due to the shock workup yielded a main effect for phase, $F(1,24)=11.60, p=.002, \eta_{\mathrm{p}}{ }^{2}=.326$, and a Phase $\times$ Fear relevance interaction, $F(1,24)=8.64, p=.007, \eta_{\mathrm{p}}{ }^{2}=.265$. The interaction reflects on larger responses to fear-relevant than nonfearrelevant animals after shock workup, $F(1,24)=4.93, p=.036$, $\eta_{\mathrm{p}}{ }^{2}=.170$, but not before, $F(1,24)=2.60, p=.120, \eta_{\mathrm{p}}{ }^{2}=.098$. The increase in responding from the last block of the first habituation sequence to the first block of the second habituation sequence was larger for the fear-relevant stimuli, $M=0.224, S D=0.288$, than for the nonfear-relevant stimuli, $M=0.111, S D=0.238$.

Electrodermal unconditional responses did not differ between the CS conditions, fear-relevant stimuli, $M=0.716, S D=0.321$, nonfear-relevant stimuli, $M=0.774, S D=0.287, \quad t(24)<1.0$, $p=.386$. The analysis of electrodermal responses during extinction (see upper panels of Figure 2) yielded no significant results, first-interval response (FIR): all $F<1.70, p>.200, \eta_{\mathrm{p}}{ }^{2}<.130$, second-interval response (SIR): all $F<1.10, p>.300, \eta_{\mathrm{p}}{ }^{2}<.070$. Five participants reported the CS-US contingencies incorrectly in the postexperimental contingency questionnaire. Analyses of the extinction data excluding these nonverbalizers yielded similar results, FIR: all $F<2.41, p>.138, \eta_{\mathrm{p}}{ }^{2}<.135$, SIR: all $F<1.90$, $p>.188, \eta_{\mathrm{p}}{ }^{2}<.092$.

Evaluative ratings. The upper panel of Figure 3 summarizes the pleasantness ratings provided for the four animal pictures that were missing for one participant. Fear-relevant animals were rated as less pleasant than nonfear-relevant animals, and conditioning effects seemed evident for nonfear-relevant stimuli, but not for fear-relevant ones. The analysis confirmed this pattern yielding main effects for fear relevance, $F(1,23)=246.58, p<.001$, $\eta_{\mathrm{p}}{ }^{2}=.915$, and phase, $F(1,23)=4.99, p=.035, \eta_{\mathrm{p}}{ }^{2}=.178$, as well as a CS $\times$ Phase interaction, $F(1,23)=10.70, p=.003, \eta_{\mathrm{p}}{ }^{2}=.317$. The Fear Relevance $\times$ CS $\times$ Phase interaction was marginal, $F(1,23)=2.95, p=.099, \eta_{\mathrm{p}}{ }^{2}=.114$. Follow-up analyses of the two-way interaction revealed no difference in the evaluation of $\mathrm{CS}^{+}$and $\mathrm{CS}^{-}$prior to conditioning, $F(1,23)=0.12, p=.737$, $\eta_{\mathrm{p}}{ }^{2}=.005$, but a marginally more negative evaluation of the $\mathrm{CS}^{+}$ after the experiment, $F(1,23)=4.06, p=.056, \eta_{\mathrm{p}}{ }^{2}=.150$. Followup comparisons of $\mathrm{CS}^{+}$and $\mathrm{CS}^{-}$on each level of the other factors confirmed lower ratings of $\mathrm{CS}^{+}$than of $\mathrm{CS}^{-}$only for the nonfearrelevant stimuli after the experiment, $F(1,23)=8.86, p=.007$, $\eta_{\mathrm{p}}{ }^{2}=.278$, all other comparisons $F<1 ., p>.680, \eta_{\mathrm{p}}{ }^{2}<.007$. Analyzing the data of verbalizers only yielded a similar pattern of results with the exception that the follow-up analysis for the twoway interaction was significant in the smaller sample: differential evaluation of $\mathrm{CS}^{+}$and $\mathrm{CS}^{-}$prior to conditioning, $F(1,18)=0.50$, $p=.488, \eta_{\mathrm{p}}{ }^{2}=.027$, after conditioning, $F(1,18)=4.70, p=.044$, $\eta_{\mathrm{p}}^{2}=.207$.

\section{Discussion}

The primary aim of Experiment 1 was to assess whether a shock workup procedure would elicit selective sensitization of electrodermal responses to pictures of animal fear-relevant stimuli relative to pictures of animal nonfear-relevant stimuli. The electrodermal data recorded in the two habituation sessions provide support for this prediction. Whereas there was no difference in electrodermal responses to animal fear-relevant and nonfear-relevant stimuli prior to the shock workup, such a difference emerged after the shock workup. Moreover, the increase in electrodermal responding from the last block of the first habituation session to the first block of the second habituation session was larger for the animal fear-relevant stimuli. This result is consistent with the results reported by Lovibond et al. (1993) and suggests that differential responding to animal fear-relevant and nonfear-relevant stimuli can be mediated by selective sensitization. 

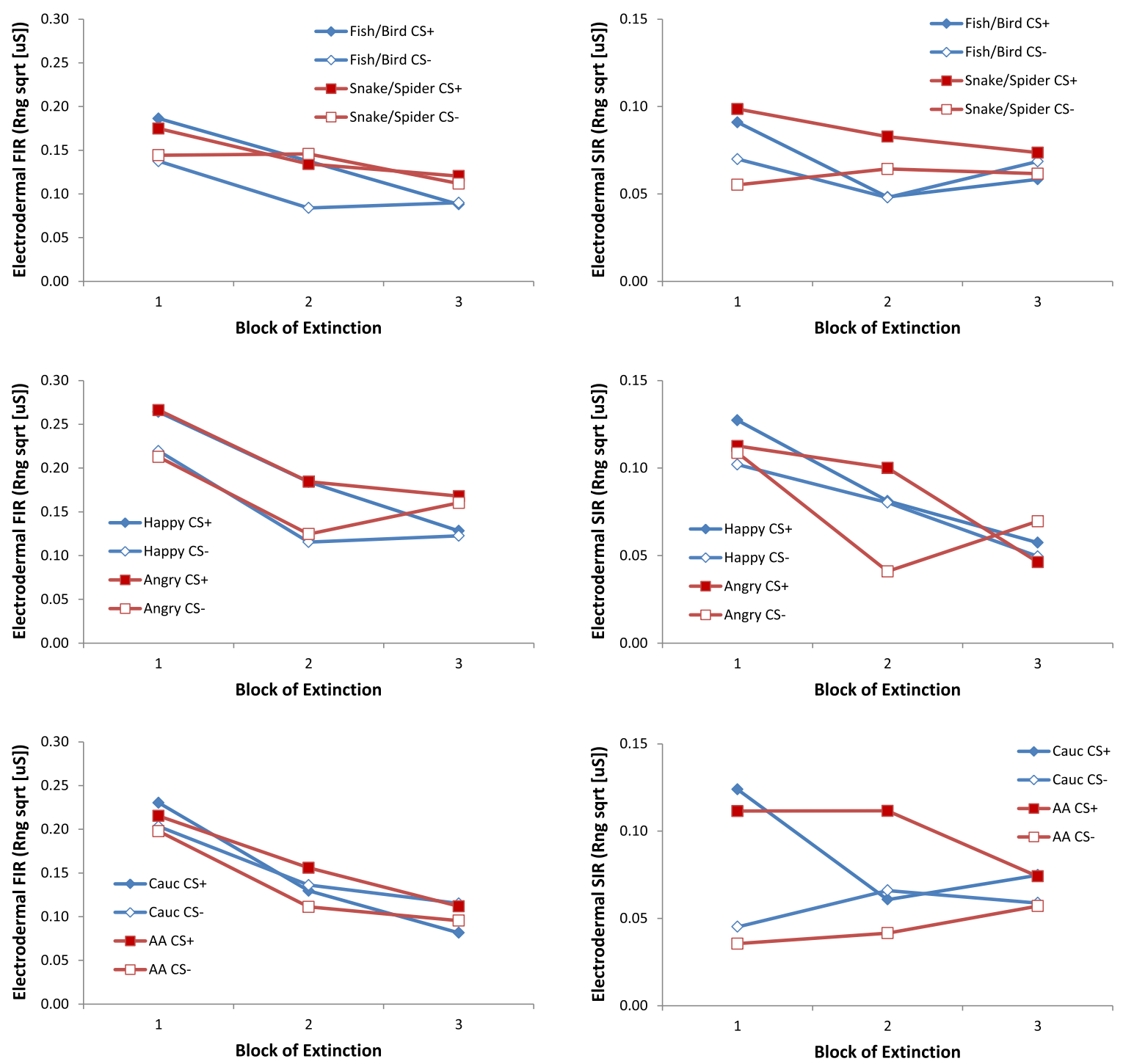

Figure 2. Mean electrodermal first-interval responses (left) and second-interval responses (right) to fear-relevant and nonfear-relevant stimuli paired with the unconditional stimulus during acquisition $\left(\mathrm{CS}^{+}\right)$or presented alone $\left(\mathrm{CS}^{-}\right)$as a function of trial blocks during extinction in Experiment 1 (upper), Experiment 2 (middle), and Experiment 3 (lower).

It should be noted that the shock workup procedure used here does not permit a conclusion as to what actually causes sensitization, be it the presentation of the electrotactile stimuli, the interaction with the experimenter, the time delay between the first and the second habituation series, the mere presence of the shock electrode, or participants' expectation that electrotactile stimuli may be presented during the second habituation sequence. However, this should not detract from the present study given that the withinsubject design means the same factors would influence responding to fear-relevant and nonfear-relevant stimuli. Given that a pattern consistent with selective sensitization was observed for animal fear-relevant stimuli in Experiment 1, the manipulation is suited to assess selective sensitization to stimuli drawn from different fear relevance domains.

A secondary aim of Experiment 1 was to replicate Öhman, Eriksson, and Olofsson's (1975) finding that fear can be conditioned to animal fear-relevant stimuli, but not to nonfear-relevant stimuli in a single trial. This prediction was not supported by the electrodermal data, which yielded no evidence for fear conditioning to either animal fear-relevant or nonfear-relevant conditional stim- uli. The rating data collected after the completion of the experiment suggest some evidence for fear learning; however, this seems to have been more pronounced for nonfear-relevant conditional stimuli. This rather unexpected finding may reflect a floor effect in the evaluations of the fear-relevant animals. Given that pictures of snakes and spiders were evaluated as rather unpleasant prior to conditioning already, it may be that a single pairing with the unconditional stimulus was not sufficient to further decrease the rated pleasantness of the fear-relevant $\mathrm{CS}^{+}$.

Experiment 2 was conducted to assess whether selective sensitization can be observed with interpersonal fear-relevant stimuli. Thus, pictures of angry and happy Caucasian faces were used as conditional stimuli instead of the animal pictures.

\section{Experiment 2}

\section{Method}

The procedure of Experiment 2 replicated that of Experiment 1 with the following exceptions. Twenty-eight undergraduate students 

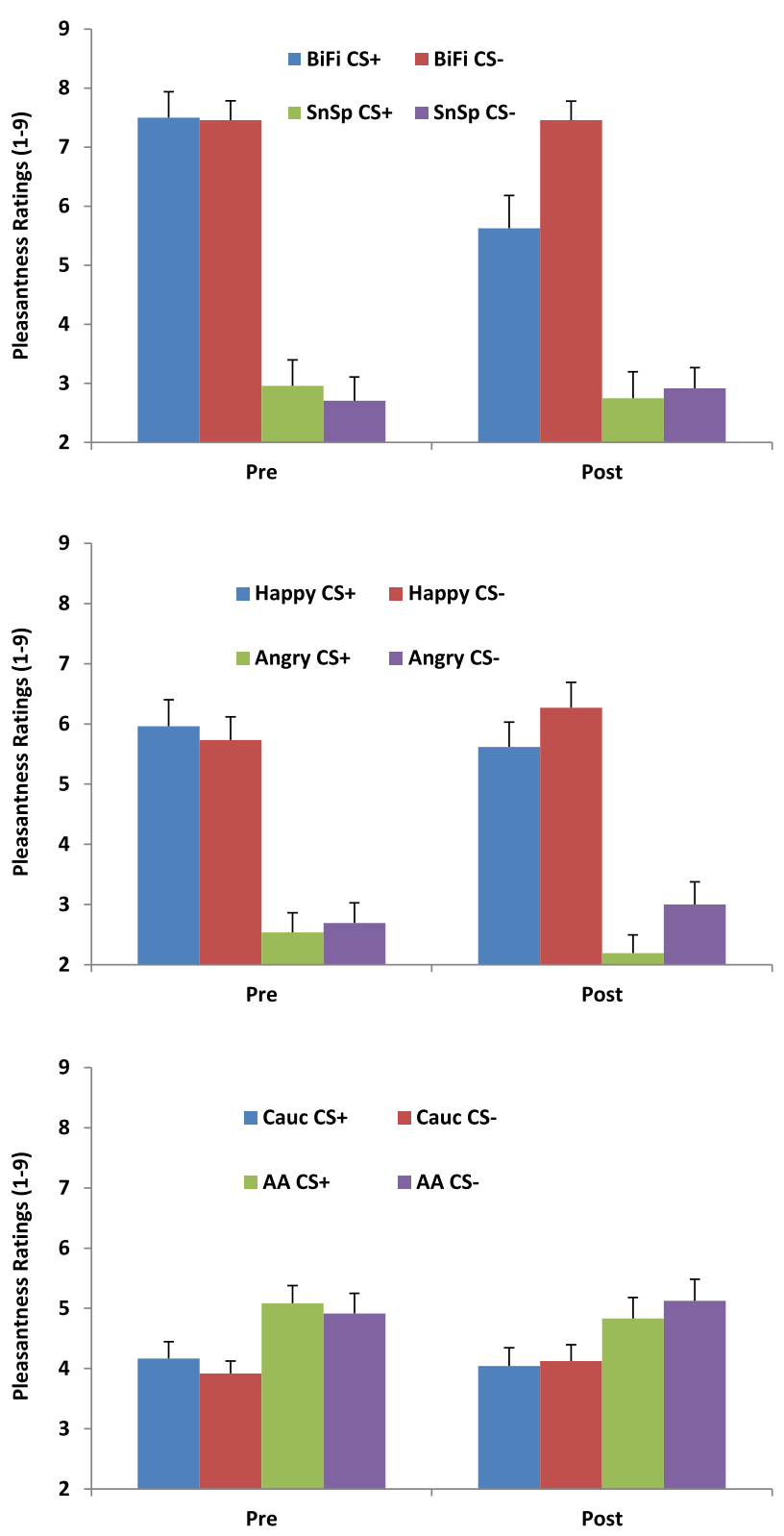

Figure 3. Pleasantness ratings for fear-relevant and nonfear-relevant stimuli provided before and after the conditioning experiment in Experiment 1 (upper), Experiment 2 (middle), and Experiment 3 (lower). Error bars represent standard errors of the means.

(10 males; 23 Caucasian, 2 South East Asian, 3 did not disclose ethnicity) aged between 17 and 37 years (mean age: 20.63 years) from Curtin University, where the research protocol had been approved by the local ethics review board, volunteered in exchange for course credit and provided informed consent. The eight colored images of animals were replaced with pictures of male Caucasian faces expressing anger or happiness (NimStim database, poses AN_O and HA_O of models 20, 21, 31, 32; Tottenham et al., 2009).

\section{Results}

Electrodermal data. The middle panel of Figure 1 displays the electrodermal first-interval responses recorded during the two habituation sequences. Electrodermal responses did not differ between fear-relevant and nonfear-relevant stimuli and declined during the first habituation phase. The shock workup procedure resulted in increased responses to both emotional expressions, with a slightly larger increase for angry faces. Subsequent habituation was fast and did not seem to differ between expressions. The omnibus ANOVA yielded main effects for phase, $F(1,27)=35.02$, $p<.001, \quad \eta_{\mathrm{p}}{ }^{2}=.565, \quad$ and block, $F(3,25)=13.75, \quad p<.001$, $\eta_{\mathrm{p}}{ }^{2}=.623$, as well as a Phase $\times$ Block interaction, $F(3,25)=5.31$, $p=.006, \eta_{\mathrm{p}}{ }^{2}=.389$. The interaction reflects on a steeper decline in electrodermal responding across blocks of habituation after the shock workup.

Electrodermal orienting at the beginning of the first habituation sequence was marginally larger to angry faces, $M=0.178$, $S D=0.136$, than to happy faces, $M=0.132, S D=0.121$, $t(27)=1.953, p=.061$. The ANOVA assessing the effect of the shock workup yielded a main effect for phase, $F(1,27)=67.24$, $p<.001, \eta_{\mathrm{p}}{ }^{2}=.713$, and a Phase $\times$ Fear Relevance interaction, $F(1,27)=5.18, p=.031, \eta_{\mathrm{p}}{ }^{2}=.161$. The interaction reflects that the shock work-up procedure resulted in a larger increase in responding from the last block of the first habituation sequence to the first block of the second habituation sequence for the angry expressions, $M=0.339, S D=0.205$, than for the happy expressions, $M=0.258, S D=0.212$. The difference in responding to angry and happy faces was marginal before, $F(1,27)=3.21$, $p=.084, \eta_{\mathrm{p}}{ }^{2}=.106$ (larger responses to happy faces), and not significant after shock workup, $F(1,27)=1.31, p=.263, \eta_{\mathrm{p}}{ }^{2}=.046$.

Electrodermal unconditional responses did not differ between the conditional stimulus conditions, angry faces, $M=0.803$, $S D=0.223$, happy faces, $M=0.858, S D=0.224, t(27)<1.10$, $p=.298$. The first and second interval electrodermal responses displayed during extinction are shown in the middle panels of Figure 2. Angry and happy $\mathrm{CS}^{+}$elicited larger first-interval responses than did the respective $\mathrm{CS}^{-}, F(1,27)=9.83, p=.004, \eta_{\mathrm{p}}{ }^{2}=.267$, and responses declined across blocks of trials, $F(2,26)=12.59$, $p<.001, \quad \eta_{\mathrm{p}}{ }^{2}=.492$. Electrodermal second-interval responses declined across blocks of trials, $F(2,26)=10.54, p<.001$, $\eta_{\mathrm{p}}{ }^{2}=.448$. Thirteen participants failed to report the CS-US contingencies correctly. Analyses of the extinction data excluding the nonverbalizers yielded similar results, FIR: main effects for CS, $F(1,14)=13.20, p=.003, \eta_{\mathrm{p}}{ }^{2}=.485$, and block, $F(2,13)=15.67$, $p<.001, \eta_{\mathrm{p}}{ }^{2}=.707 ;$ SIR: main effects for fear relevance, $F(1,14)=7.73, p=.015, \eta_{\mathrm{p}}{ }^{2}=.356$ (angry faces, $M=0.063$, $S D=0.096$, happy faces, $M=0.091, S D=0.107$; happy $>$ angry), and block, $F(2,13)=8.91, p=.004, \eta_{\mathrm{p}}{ }^{2}=.578$.

Evaluative ratings. The middle panel of Figure 3 summarizes the pleasantness ratings provided by 26 of the participants prior to and after conditioning. Angry faces were rated as less pleasant than happy faces, $F(1,25)=39.01, p<.001, \eta_{\mathrm{p}}{ }^{2}=.609$, and angry and happy $\mathrm{CS}^{+}$seemed to be rated as less pleasant than the respective $\mathrm{CS}^{-}$after the experiment; however, this was only supported by a marginal CS $\times$ Phase interaction, $F(1,25)=4.04, p=.055$, $\eta_{\mathrm{p}}{ }^{2}=.139$. Follow-up analyses of the marginal interaction revealed a significant difference between the CSs after, $F(1,25)=4.47$, $p=.045, \eta_{\mathrm{p}}{ }^{2}=.152$, but not before conditioning, $F(1,25)<1$, $p>$.80. Analysis of the data from verbalizers only provided a similar pattern of results.

\section{Discussion}

The results of Experiment 2 are consistent with the notion that interpersonal fear-relevant stimuli are subject to selective 
sensitization. Although there was no difference in electrodermal responding between angry and happy faces at the onset of the second habituation sequence, the increase in responding from the end of habituation Sequence 1 to the beginning of habituation Sequence 2 was larger for angry than for happy faces. In contrast to Experiment 1 , there was evidence for one-trial fear conditioning in electrodermal first-interval responses. However, contrary to the predictions of the prepared learning account, this finding emerged for angry and for happy facial expressions. The rating data confirm this result indicating differential evaluations of $\mathrm{CS}^{+}$and $\mathrm{CS}^{-}$after conditioning regardless of the fear relevance of the facial expressions of emotion.

Experiment 3 was conducted to assess whether selective sensitization can be observed with intergroup fear-relevant stimuli. To address this question, pictures of African American and Caucasian faces were used as conditional stimuli in a group of Caucasian participants.

\section{Experiment 3}

\section{Method}

Experiment 3 replicated the procedure used in Experiment 1 with the following exceptions. Twenty-five Caucasian undergraduate students (10 males) aged between 19 and 43 years (mean age: 23.96 years) volunteered in exchange for course credit and provided informed consent. The research protocol had been approved by the University of Queensland ethics review board. The eight colored images of animals were replaced with pictures of male African American and Caucasian faces with neutral facial expressions (NimStim database, poses NE_C of models 20, 21, 31, 32, 38, 40, 42, and 43; Tottenham et al., 2009).

\section{Results}

Electrodermal data. The lower panel of Figure 1 displays the electrodermal first-interval responses recorded during the two habituation sequences. Electrodermal responses were larger after shock workup, $F(1,24)=35.47, p<.001, \eta_{\mathrm{p}}{ }^{2}=.596$, and declined across blocks of trials, $F(3,22)=12.53, p<.001, \eta_{\mathrm{p}}{ }^{2}=.631$; however, this decline was more pronounced in the second habituation sequence, $F(3,22)=4.70, p=.011, \eta_{\mathrm{p}}{ }^{2}=.390$. The omnibus analysis also yielded a trend toward a three-way interaction, $F(3,22)=2.43, p=.093, \eta_{\mathrm{p}}{ }^{2}=.249$. This trend reflects that African American faces elicited larger electrodermal responses than Caucasian faces in Blocks 1 and 4 of the second habituation sequence, both $F(1,24)>4.65, p<.045, \eta_{\mathrm{p}}{ }^{2}>.160$, whereas the other comparisons were not significant, all $F(1,24)<1.60$, $p>.220, \eta_{\mathrm{p}}^{2}<.045$.

African American faces did not elicit larger electrodermal orienting at the beginning of the first habituation sequence, $M=0.101, \quad S D=0.128$, than Caucasian faces, $M=0.121$, $S D=0.125, t(24)<1.0, p=.471$. The targeted ANOVA assessing sensitization effects yielded main effects for phase, $F(1,24)=51.11, \quad p<.001, \quad \eta_{\mathrm{p}}{ }^{2}=.680, \quad$ and fear relevance, $F(1,24)=5.04, p=.034, \eta_{\mathrm{p}}{ }^{2}=.174$, with larger responses to African American faces. The Phase $\times$ Fear Relevance interaction was not significant on the preset level, $F(1,24)=2.58, p=.122$, $\eta_{\mathrm{p}}{ }^{2}=.097$. Inspection of the lower panel of Figure 1 suggests that the difference in responding to Caucasian and African American faces was evident more strongly after the shock workup. This impression was confirmed by supplementary analyses. Responses to African American faces were larger than to Caucasian faces after the workup, $F(1,24)=4.69, p=.040, \eta_{\mathrm{p}}{ }^{2}=.164$, but not before, $F(1,24)=0.08, p=.774, \eta_{\mathrm{p}}^{2}=.003$.

Electrodermal unconditional responses did not differ between the CS conditions: African American faces, $M=0.897$, $S D=0.154$, Caucasian faces, $M=0.888, S D=0.151, t(24)<1.0$, $p=.804$. The first- and second-interval electrodermal responses displayed during extinction are shown in the lower panels of Figure 2. First-interval responses to African American and Caucasian faces declined across blocks of trials, $F(2,23)=10.48, p<.001$, $\eta_{\mathrm{p}}{ }^{2}=.477$. Second-interval responses were larger to $\mathrm{CS}^{+}$than to $\mathrm{CS}^{-}, \quad F(1,24)=8.22, \quad p=.008, \quad \eta_{\mathrm{p}}{ }^{2}=.255$, a difference that declined across blocks, CS $\times$ Block interaction, $F(2,23)=4.17$, $p=.029, \eta_{\mathrm{p}}{ }^{2}=.266$. The difference between $\mathrm{CS}^{+}$and $\mathrm{CS}^{-}$was significant at the first block of extinction, $F(1,24)=12.40$, $p=.002, \eta_{\mathrm{p}}{ }^{2}=.341$, but not at later blocks, both $F<2.80, p>.10$, $\eta_{\mathrm{p}}{ }^{2}<.11$.

Three participants failed to report the CS-US contingencies correctly. Analyses of the extinction data excluding the nonverbalizers yielded similar if somewhat weaker results, FIR: main effects for block, $F(2,20)=7.18, p=.004, \eta_{\mathrm{p}}{ }^{2}=.418$; SIR: main effects for $\mathrm{CS}, F(1,21)=7.01, p=.015, \eta_{\mathrm{p}}{ }^{2}=.250, \mathrm{CS} \times$ Block interaction, $F(2,20)=4.38, p=.027, \eta_{\mathrm{p}}^{2}=.304$.

Evaluative ratings. The lower panel of Figure 3 summarizes the pleasantness ratings provided for African American and Caucasian faces prior to and after conditioning training by 24 of the participants. Caucasian faces were rated as less pleasant than African American faces, $F(1,23)=14.92, p=.001, \eta_{\mathrm{p}}{ }^{2}=.393$. Analysis of the data including only the participants who could verbalize the contingencies provided a similar pattern of results, main effect for fear relevance, $F(1,20)=12.13, p=.002, \eta_{\mathrm{p}}{ }^{2}=.377$.

\section{Discussion}

The results provided by Experiment 3 are consistent with those of the previous experiments. Electrodermal responses to African American and Caucasian faces did not differ before the sensitizing shock workup procedure, but responses to African American faces exceeded those to Caucasian faces after the workup. However, contrary to Experiment 2, the targeted analysis yielded no significant interaction. Thus, the current results need to be interpreted with caution and require further replication. Similar to Experiment 2, Experiment 3 revealed evidence for one-trial fear learning that was consistent across both fear relevance conditions. For African American and for Caucasian faces, second-interval electrodermal responses were larger to $\mathrm{CS}^{+}$than to $\mathrm{CS}^{-}$.

The rating data for Experiment 3 deviate from those obtained in Experiments 1 and 2 in that the fear-relevant out-group faces were rated as more positive than the nonfear-relevant in-group faces. This finding is unexpected, but consistent with rating data reported by Bramwell et al. (2014) for happy African American and Caucasian faces. The positive explicit evaluations of out-group faces may reflect the participant's desire to appear free of prejudice. Future studies using these stimulus materials should employ implicit measures of stimulus evaluation that can undercut such tendencies toward socially desirable behavior (Greenwald, McGhee, \& Schwartz, 1998). 


\section{General Discussion}

The main objective of the present study was to assess whether, relative to nonfear-relevant stimuli, selective sensitization is observed in electrodermal responding to animal, interpersonal, and intergroup fear-relevant stimuli (see Lovibond et al., 1993). Sensitization was induced using a shock workup procedure in which participants were asked to set the intensity of an electrotactile unconditional stimulus to a level they perceived as unpleasant, but not painful. In this procedure, electrotactile stimuli of increasing intensity were presented repeatedly until such an intensity level was reached. The secondary objective was to assess whether onetrial fear learning would be observed to the fear-relevant but not the nonfear-relevant conditional stimuli used across the three experiments (Öhman et al., 1975).

The pattern of results provided by the three experiments is rather consistent across stimulus domains. Selective sensitization of electrodermal responses was observed for animal, interpersonal, and intergroup fear-relevant stimuli. Electrodermal responses to snakes and spiders, angry faces, and African American faces did not differ significantly from responses to their respective control stimuli, fish and birds, happy faces, or Caucasian faces upon first encounter, and a marginal difference emerged for interpersonal fear-relevant stimuli only. Snakes and spiders and African American faces elicited larger electrodermal responses than did their respective controls after the shock workup procedure had been completed. This difference was not significant for the interpersonal fear-relevant stimuli; however, responses to angry faces increased more from before to after the shock workup than did responses to happy faces. ${ }^{1}$ The finding of enhanced sensitization to fear-relevant stimuli across all stimulus domains complements the findings of enhanced resistance to extinction that have been documented for these stimuli (Öhman et al., 1976; Öhman \& Dimberg, 1978; Olsson et al., 2005).

The current study provides evidence for selective sensitization across domains of fear-relevant stimuli; however, it is not diagnostic as to the mechanism that mediates this difference between fearrelevant and nonfear-relevant stimuli. Evolutionary-mediated fear relevance has been offered to explain enhanced responding to snakes and interpersonal fear-relevant stimuli (Öhman, 2009; Öhman, Soares, Juth, Lindström, \& Esteves, 2012), but has been questioned as suitable to explain enhanced responding to spiders (Öhman et al., 2012) or to intergroup fear-relevant stimuli (Olsson et al., 2005). The latter assertion is based mainly on the consideration that distinct races are a relatively recent phenomenon having developed within the past 100,000-200,000 years (Molnar, 2006; but see Hawks, Wang, Cochran, Harpending, \& Moyzis, 2007, for a different view on human adaptive evolution). The notion that

1. In order to confirm the reliability of the pattern of results across experiments, we repeated the targeted analyses including a betweensubject factor experiment. The assessment of the responses at the beginning of the first habituation sequence yielded no significant results, all $F<1.10, p>.360$. The ANOVA assessing the effect of the shock workup yielded a main effect for phase, $F(1,75)=103.90, p<.001$, $\eta_{\mathrm{p}}{ }^{2}=.581$, and a Phase $\times$ Fear Relevance interaction, $F(1,75)=14.72$, $p<.001, \eta_{\mathrm{p}}{ }^{2}=.164$. The interaction reflects that responses to fearrelevant stimuli $(M=0.064, S D=0.113)$ were marginally smaller than response to nonfear-relevant stimuli $(M=0.093, S D=0.148)$ before the shock workup, $F(1,75)=3.65, p=.060, \eta_{\mathrm{p}}{ }^{2}=.046$, but larger after the workup $(M=0.349, \quad S D=0.209 \quad$ vs. $M=0.287, \quad S D=0.183)$, $F(1,75)=11.02, p=.001, \eta_{\mathrm{p}}{ }^{2}=.128$. The largest term involving the factor experiment was a marginal Experiment $\times$ Phase interaction, $F(2,75)=2.71, p=.073, \eta_{\mathrm{p}}{ }^{2}=.067$, with all other terms not significant, $F<1.90, p>.160$. responses to spiders do not reflect evolutionary-mediated fear relevance is derived from the difficulty to construct a strong argument in support of a selection advantage for ancestors of homo sapiens that readily developed a fear of spiders. Unlike snakes, spiders did not prey on our evolutionary ancestors (Öhman, 2009; Öhman et al., 2012).

Lovibond et al. (1994) offered a cognitive model to account for selective sensitization effects observed to ontogenetic fear-relevant stimuli like pointed guns (see also Hugdahl \& Johnsen, 1989). Consistent with the expectancy model proposed by Davey (1992), these authors argue that enhanced responding to fear-relevant stimuli under conditions of threat may reflect an enhanced expectation that presentations of these stimuli may be followed by shock. Davey (1992) found these differential expectations to be evident in online ratings of unconditional stimulus expectancy a priori. They were expressed in differential electrodermal responses between animal fear-relevant and nonfear-relevant stimuli only after the threat, a tactile/acoustic unconditional stimulus compound, had actually been experienced. These differential expectations can be rooted in a variety of sources, real or vicarious experiences, information provided, or negative stereotypes and social norms (Olsson et al., 2005). The latter may be of particular importance in the context of interpersonal and intergroup fear relevance.

It should be noted, of course, that observing the same patterns of results for animal, interpersonal, and intergroup fear-relevant stimuli in response to extinction and sensitization procedures does not imply a single underlying mechanism. The observation that effects of selective sensitization are short lasting (see Figure 1) whereas resistance to extinction can be observed across numerous blocks of extinction trials has been cited to support a distinction between selective sensitization and selective associations (Öhman $\&$ Mineka, 2001). Moreover, dissociations across stimulus domains have been shown - fear conditioned to animal fear-relevant stimuli is encapsulated from cognition, whereas fear conditioned to interpersonal and intergroup fear-relevant stimuli is not (for a review, see Mallan et al., 2013). This overall pattern of results suggests that the concept of fear relevance as discussed in the literature is complex and currently not well understood. More empirical work is required to map the similarities and differences across the domains of fear relevance in order to gain a better understanding of the mechanism(s) that these findings reflect.

A secondary aim of the present study was to assess whether one-trial fear learning would be observed in the current procedures and limited to the fear-relevant stimuli drawn from the different stimulus domains. This was not the case. Differential electrodermal fear learning after a single pairing of conditional and unconditional stimulus was evident in Experiments 2 and 3, but not in Experiment 1 . Differential evaluations of the conditional stimuli based on self-report were evident in Experiments 1 (significant if only verbalizers were considered) and 2, but not in Experiment 3. Most important in the present context, if differential fear learning was evident, it was found for fear-relevant and nonfear-relevant conditional stimuli, a finding that is inconsistent with the prediction of preparedness theory (Seligman, 1971) or prior reports (Öhman et al., 1975). Thus, although the present procedure was suited to support one-trial fear learning, this learning was not selective to fear-relevant conditional stimuli.

It should be noted, however, that the current procedure differed considerably from that employed by Öhman et al. (1975). The latter used a completely between-subject design with a single cue conditioning procedure whereas a completely within-subject design was employed in the present study. Although there is 
evidence for selective one-trial learning in a differential conditioning procedure (see Davey, 1992, Experiment 4), using a within-subject design in which two $\mathrm{CS}^{+}$were paired with one US each may have supported fear learning to the nonfear-relevant stimuli, reducing the difference between stimulus conditions. Taken together, it seems that confirmation as to whether interpersonal and intergroup fear-relevant stimuli fulfill the one-trial learning criterion postulated by preparedness theory is still pending.
In summary, the current study was designed to assess whether selective sensitization would be observed in response to animal, interpersonal, and intergroup fear-relevant stimuli. This prediction was confirmed in that, after a shock workup procedure, responding to animal and intergroup fear-relevant stimuli exceeded that to their respective controls and the increase in responding was larger to interpersonal fear-relevant stimuli than to their controls. This consistent pattern of results supports the notion of similarities in stimulus processing across different domains of fear relevance.

\section{References}

Bond, N. W., \& Siddle, D. A. T. (1986). The preparedness account of social phobia: Some data and alternative explanations. In R. M. Rapee (Ed.), Current controversies in anxiety disorders (pp. 291-316). New York, NY: Guilford.

Bramwell, S., Mallan, K. M., \& Lipp, O. V. (2014). Are two threats worse than one? The effects of face race and emotion on fear conditioning. Psychophysiology, 51, 152-158. doi: 10.1111/psyp.12155

Davey, G. C. L. (1992). An expectancy model of laboratory preparedness effects. Journal of Experimental Psychology: General, 121, 24-40. doi: 10.1037/0096-3445.121.1.24

Esteves, F., Dimberg, U., \& Öhman, A. (1994). Automatically elicited fear: Conditioned skin conductance responses to masked facial expressions. Cognition and Emotion, 8, 393-413. doi: 10.1080/02699939408408949

Forster, K. I., \& Forster, J. C. (2003). DMDX: A Windows display program with millisecond accuracy. Behavior Research Methods, Instruments \& Computers, 35, 116-124. doi: 10.3758/BF03195503

Greenwald, A. G., McGhee, D. E., \& Schwartz, J. L. K. (1998). Measuring individual differences in implicit cognition: The implicit association test. Journal of Personality and Social Psychology, 74, 1464-1480. doi: 10.1037/0022-3514.74.6.1464

Hamm, A. O., Vaitl, D., \& Lang, P. J. (1989). Fear conditioning, meaning, and belongingness: A selective association analysis. Journal of Abnormal Psychology, 98, 395-406. doi: 10.1037/0021-843X.98.4.395

Hawks, J., Wang, E. T., Cochran, G. M., Harpending, H. C., \& Moyzis, R. K. (2007). Recent acceleration of human adaptive evolution. Proceedings of the National Academy of Sciences of the USA, 104, 20753-20758. doi: 10.1073/pnas.0707650104

Ho, Y., \& Lipp, O. V. (2014). Faster acquisition of conditioned fear to fear-relevant than to non fear-relevant conditional stimuli. Psychophysiology, 51, 810-813. doi: 10.1111/psyp.12223

Hugdahl, K., \& Johnsen, B. H. (1989). Preparedness and electrodermal fear-conditioning: Ontogenetic vs phylogenetic explanations. Behaviour, Research and Therapy, 27, 269-278. doi: 10.1016/00057967(89)90046-6

Lanzetta, J. T, \& Orr, S. P. (1980). Influence of facial expressions on the classical conditioning of fear. Journal of Personality and Social Psychology, 39, 1081-1087. doi: 10.1037/h0077713

Lanzetta, J. T, \& Orr, S. P. (1981). Stimulus properties of facial expressions and their influence on the classical conditioning of fear. Motivation and Emotion, 5, 225-234. doi: 10.1007/BF00993886

Lovibond, P. F., Hanna, S. K., Siddle, D. A. T., \& Bond, N. W. (1994). Electrodermal and subjective reactions to fear-relevant stimuli under threat of shock. Australian Journal of Psychology, 46, 73-80. doi: $10.1080 / 00049539408259476$

Lovibond, P. F., Siddle, D. A. T., \& Bond, N. W. (1993). Resistance to extinction of fear-relevant stimuli: Preparedness or selective sensitization? Journal of Experimental Psychology: General, 122, 449-461. doi: 10.1037/0096-3445.122.4.449

Mallan, K. M., Lipp, O. V., \& Cochrane, B. (2013). Slithering snakes, angry men and out-group members: What and whom are we evolved to fear? Cognition \& Emotion, 27, 1168-1180. doi: 10.1080/ 02699931.2013.778195

Mazurski, E. J., Bond, N. W., Siddle, D. A. T., \& Lovibond, P. F. (1996). Conditioning with facial expressions of emotion: Effects of CS sex and age. Psychophysiology, 33, 416-425. doi: 10.1111/j.1469-8986. 1996.tb01067.x

Molnar, S. (2006). Human variation: Races, types, and ethnic groups (6th ed.). Upper Saddle River, NJ: Pearson Prentice Hall.
Öhman, A. (1983). The orienting response during Pavlovian conditioning. In D. A. T. Siddle (Ed.), Orienting and habituation: Perspectives in human research (pp. 315-370). New York, NY: Wiley.

Öhman, A. (2009). Of snakes and faces: An evolutionary perspective on the psychology of fear. Scandinavian Journal of Psychology, 50, 543552. doi: $10.1111 / \mathrm{j} .1467-9450.2009 .00784 . x$

Öhman, A., \& Dimberg, U. (1978). Facial expressions as conditioned stimuli for electrodermal responses: A case of "preparedness"? Journal of Personality and Social Psychology, 36, 1251-1258. doi: 10.1037/00223514.36.11.1251

Öhman, A., Eriksson, A., \& Olofsson, C. (1975). One-trial learning and superior resistance to extinction of autonomic responses conditioned to potentially phobic stimuli. Journal of Comparative and Physiological Psychology, 88, 619-627. doi: 10.1037/h0078388

Öhman, A., Eriksson, A., Fredriksson, M., Hugdahl, K., \& Olofsson, C. (1974). Habituation of the electrodermal orienting reaction to potentially phobic and supposedly neutral stimuli in normal human subjects. Biological Psychology, 2, 85-93.

Öhman, A., Fredrikson, M., Hugdahl, K., \& Rimmö, P. (1976). The premise of equipotentiality in human classical conditioning: Conditioned electrodermal responses to potentially phobic stimuli. Journal of Experimental Psychology: General, 105, 313-337. doi: 10.1037/00963445.105.4.313

Öhman, A., \& Mineka, S. (2001). Fears, phobias, and preparedness: Toward an evolved module of fear and fear learning. Psychological Review, 108, 483-522. doi: 10.1037/0033-295X.108.3.483

Öhman, A., Soares, S. C., Juth, P., Lindström, B., \& Esteves, F. (2012). Evolutionary derived modulations of attention to two common fear stimuli: Serpents and hostile humans. Journal of Cognitive Psychology, 24, 17-32. doi: 10.1080/20445911.2011.629603

Olsson, A., Ebert, J. P., Banaji, M. R., \& Phelps, E. A. (2005). The role of social groups in the persistence of learned fear. Science, 309, 785-787. doi: $10.1126 /$ science. 1113551

Orr, S. P., \& Lanzetta, J. T. (1980). Facial expressions of emotion as conditioned stimuli for human autonomic responses. Journal of Personality and Social Psychology, 38, 278-282. doi: 10.1037/0022-3514.38.2.278

Parra, C., Esteves, F., Flykt, A., \& Öhman, A. (1997). Pavlovian conditioning to social stimuli: Backward masking and the dissociation of implicit and explicit cognitive processes. European Psychologist, 2, 106-117. doi: 10.1027/1016-9040.2.2.106

Pitman, R. K., \& Orr, S. P. (1986). Test of the conditioning model of neurosis: Differential aversive conditioning of angry and neutral facial expressions in anxiety disorder patients. Journal of Abnormal Psychology, 95, 208-213. doi: 10.1037/0021-843X

Prokasy, W. F., \& Kumpfer, K. L. (1973). Classical conditioning. In W. F. Prokasy \& D. C. Raskin (Eds.), Electrodermal activity in psychological research (pp. 157-202). San Diego, CA: Academic Press.

Rowles, M. E., Lipp, O. V., \& Mallan, K. M. (2012). On the resistance to extinction of fear conditioned to angry faces. Psychophysiology, 49, 375-380. doi: 10.1111/j.1469-8986.2011.01308.x

Seligman, M. E. P. (1971). Phobias and preparedness. Behavior Therapy, 2, 307-320. doi: 10.1016/S0005-7894(71)80064-3

Tottenham, N., Tanaka, J. W., Leon, A. C., McCarry, T., Nurse, M., Hare, T. A., ... Nelson, C. (2009). The NimStim set of facial expressions: Judgments from untrained research participants. Psychiatry Research, 168, 242-249. doi: 10.1016/j.psychres.2008.05.006

(Received November 19, 2014; AcCePted July 17, 2015) 